\title{
CENTRAL QUOTIENT VERSUS COMMUTATOR SUBGROUP OF GROUPS
}

\author{
MANOJ K. YADAV
}

\begin{abstract}
In 1904, Issai Schur proved the following result. If $G$ is an arbitrary group such that $G / \mathrm{Z}(G)$ is finite, where $\mathrm{Z}(G)$ denotes the center of the group $G$, then the commutator subgroup of $G$ is finite. A partial converse of this result was proved by B. H. Neumann in 1951. He proved that if $G$ is a finitely generated group with finite commutator subgroup, then $G / \mathrm{Z}(G)$ is finite. In this short note, we exhibit few arguments of Neumann, which provide further generalizations of converse of the above mentioned result of Schur. We classify all finite groups $G$ such that $|G / \mathrm{Z}(G)|=\left|\gamma_{2}(G)\right|^{d}$, where $d$ denotes the number of elements in a minimal generating set for $G / \mathrm{Z}(G)$. Some problems and questions are posed in the sequel.
\end{abstract}

\section{INTRODUCTION}

In 1951, Neumann [18, Theorem 5.3] proved the following result: If the index of $\mathrm{Z}(G)$ in $G$ is finite, then $\gamma_{2}(G)$ is finite, where $\mathrm{Z}(G)$ and $\gamma_{2}(G)$ denote the center and the commutator subgroup of $G$ respectively. He mentioned [19, End of page 237] that this result can be obtained from an implicit idea of Schur [23, and his proof also used Schur's basic idea. However there is no mention of this fact in [18 in which Schur's paper is also cited. In this note, this result will be termed as 'the Schur's theorem'. Neumann also provided a partial converse of the Schur's theorem [18, Corollary 5.41] as follows: If $G$ is finitely generated by $k$ elements and $\gamma_{2}(G)$ is finite, then $G / \mathrm{Z}(G)$ is finite, and bounded by $|G / \mathrm{Z}(G)| \leq\left|\gamma_{2}(G)\right|^{k}$.

Our first motivation of writing this note is to exhibit an idea of Neumann [18, page 179] which proves much more than what is said above on converse of the Schur's theorem. We quote the text here (with a minor modification in the notations):

"Let $G$ be generated by $g_{1}, g_{2}, \ldots, g_{k}$. Then

$$
\mathrm{Z}(G)=\cap_{\kappa=1}^{\kappa=k} C_{G}\left(g_{\kappa}\right) ;
$$

for, an element of $G$ lies in the centre if and only if it (is permutable) commutes with all the generators of $G$. If $G$ is an FC-group (group whose all conjugacy classes are of finite length), then $\left|G: C_{G}\left(g_{\kappa}\right)\right|$ is finite for $1 \leq \kappa \leq k$, and $\mathrm{Z}(G)$, as intersection of a finite set of subgroups of finite index, also has finite index. The index of the intersection of two subgroups does not exceed the product of the indices of the subgroups: hence in this case one obtains an upper bound for the index of the centre, namely

$$
|G: \mathrm{Z}(G)| \leq \Pi_{\kappa=1}^{\kappa=k}\left|G: C_{G}\left(g_{\kappa}\right)\right| . "
$$

Just a soft staring at the quoted text for a moment or two suggests the following. The conclusion does not require the group $G$ to be FC-group. It only requires the finiteness of the conjugacy classes of the generating elements. If a generator of the group $G$ is contained in $\mathrm{Z}(G)$, one really does not need to count it. Thus the argument works perfactly well even if $G$ is

2010 Mathematics Subject Classification. Primary 20F24, 20E45.

Key words and phrases. commutator subgroup, Schur's theorem, class-preserving automorphism. 
generated by infinite number of elements, all but finite of them lie in the center of $G$. Thus the following result holds true.

Theorem A. Let $G$ be an arbitrary group such that $G / \mathrm{Z}(G)$ is finitely generated by $x_{1} \mathrm{Z}(G)$, $x_{2} \mathrm{Z}(G), \ldots, x_{t} \mathrm{Z}(G)$ and the conjugacy class of $x_{i}$ in $G$ is of finite length for $1 \leq i \leq t$. Then $G / \mathrm{Z}(G)$ is finite. Moreover $|G / \mathrm{Z}(G)| \leq \Pi_{i=1}^{t}\left|x_{i}^{G}\right|$ and $\gamma_{2}(G)$ is finite, where $x_{i}^{G}$ denotes the conjugacy class of $x_{i}$ in $G$.

Neumann's result [18, Corollary 5.41] was reproduced by Hilton [12, Theorem 1]. It seems that Hilton was not aware of Neumann's result. This lead two more publications [21] and 24] dedicated to proving special cases of Theorem A.

Converse of the Schur's theorem is not true in general as shown by infinite extraspecial $p$ groups, where $p$ is an odd prime. It is interesting to know that example of such a 2-group also exists, which is mentioned on page 238 (second para of Section 3) of [19]. It is a central product of infinite copies of quaternion groups of order 8 amalgamated at the center of order 2 .

Our second motivation of writing this note is to provide a modification of an innocent looking result of Neumann [20, Lemma 2], which allows us to say little more on converse of the Schur's theorem. A modified version of this lemma is the following.

Lemma 1.1. Let $G$ be an arbitrary group having a normal abelian subgroup $A$ such that the index of $C_{G}(A)$ in $G$ is finite and $G / A$ is finitely generated by $g_{1} A, g_{2} A, \ldots, g_{r} A$, where $\left|g_{i}^{G}\right|<\infty$ for $1 \leq i \leq r$. Then $G / Z(G)$ is finite.

This lemma helps proving the first three statements of the following result.

Theorem B. For an arbitrary group $G, G / \mathrm{Z}(G)$ is finite if any one of the following holds true:

(i) $\mathrm{Z}_{2}(G) / \mathrm{Z}\left(\mathrm{Z}_{2}(G)\right)$ is finitely generated and $\gamma_{2}(G)$ is finite.

(ii) $G / \mathrm{Z}\left(\mathrm{Z}_{2}(G)\right)$ is finitely generated and $G /\left(\mathrm{Z}_{2}(G) \gamma_{2}(G)\right)$ is finite.

(iii) $\gamma_{2}(G)$ is finite and $\mathrm{Z}_{2}(G) \leq \gamma_{2}(G)$.

(iv) $\gamma_{2}(G)$ is finite and $G / \mathrm{Z}(G)$ is purely non-abelian.

Our final motivation is to provide a classification of all groups $G$ upto isoclinism (see Section 3 for the definition) such that $|G / \mathrm{Z}(G)|=\left|\gamma_{2}(G)\right|^{d}$ is finite, where $d$ denotes the number of elements in a minimal generating set for $G / \mathrm{Z}(G)$, discuss example in various situations and pose some problems. We conclude this section with fixing some notations. For an arbitrary group $G$, by $\mathrm{Z}(G), \mathrm{Z}_{2}(G)$ and $\gamma_{2}(G)$ we denote the center, the second center and the commutator subgroup of $G$ respectively. For $x \in G,[x, G]$ denotes the set $\{[x, g] \mid g \in G\}$. Notice that $|[x, G]|=\left|x^{G}\right|$, where $x^{G}$ denotes the conjugacy class of $x$ in $G$. If $[x, G] \subseteq \mathrm{Z}(G)$, then $[x, G]$ becomes a subgroup of $G$. For a subgroup $H$ of $G, \mathrm{C}_{G}(H)$ denotes the centralizer of $H$ in $G$ and for an element $x \in G, \mathrm{C}_{G}(x)$ denotes the centralizer of $x$ in $G$.

\section{PROOFS}

We start with the proof of Lemma 1.1, which is essentialy same as the one given by Neumann. Proof of Lemma 1.1. Let $G / A$ be generated by $g_{1} A, g_{2} A, \ldots, g_{r} A$ for some $g_{i} \in G$, where $1 \leq i \leq r<\infty$. Let $X:=\left\{g_{1}, g_{2}, \ldots, g_{r}\right\}$ and $A$ be generated by a set $Y$. Then $G=\langle X \cup Y\rangle$ and $\mathrm{Z}(G)=\mathrm{C}_{G}(X) \cap C_{G}(Y)$. Notice that $C_{G}(A)=C_{G}(Y)$. Since $C_{G}(A)$ is of finite index, $C_{G}(Y)$ is also of finite index in $G$. Also, since $\left|g_{i}^{G}\right|<\infty$ for $1 \leq i \leq r, C_{G}(X)$ is of finite index in $G$. Hence the index of $Z(G)$ in $G$ is finite and the proof is complete. 
Proof of Theorem A can be made quite precise by using Lemma 1.1.

Proof of Theorem A. Taking $A=\mathrm{Z}(G)$ in Lemma 1.1, it follows that $G / \mathrm{Z}(G)$ is finite. Moreover,

$$
|G / \mathrm{Z}(G)|=\left|G / \cap_{i=1}^{t} C_{G}\left(x_{i}\right)\right| \leq \Pi_{i=1}^{t}\left|G: C_{G}\left(x_{i}\right)\right|=\Pi_{i=1}^{t}\left|\left[x_{i}, G\right]\right|=\Pi_{i=1}^{t}\left|x_{i}^{G}\right| .
$$

That $\gamma_{2}(G)$ is finite now follows from the Schur's theorem.

For the proof of Theorem B we need the following result of Hall [9] and the subsequent proposition.

Theorem 2.1. If $G$ is an arbitrary group such that $\gamma_{2}(G)$ is finite, then $G / \mathrm{Z}_{2}(G)$ is finite.

Explicit bounds on the order of $G / \mathrm{Z}_{2}(G)$ were first given by Macdonald [14, Theorem 6.2] and later on improved by Podoski and Szegedy [22] by showing that if $\left|\gamma_{2}(G) /\left(\gamma_{2}(G) \cap \mathrm{Z}(G)\right)\right|=n$, then $\left|G / \mathrm{Z}_{2}(G)\right| \leq n^{c \log _{2} n}$ with $c=2$.

Proposition 2.2. Let $G$ be an arbitrary group such that $\gamma_{2}(G)$ is finite and $G / \mathrm{Z}(G)$ is infinite. Then $G / \mathrm{Z}(G)$ has an infinite abelian group as a direct factor.

Proof. Since $\gamma_{2}(G)$ is finite, by Theorem $2.1 G / \mathrm{Z}_{2}(G)$ is finite. Thus $\mathrm{Z}_{2}(G) / \mathrm{Z}(G)$ is infinite. Again using the finiteness of $\gamma_{2}(G)$, it follows that the exponent of $\mathrm{Z}_{2}(G) / \mathrm{Z}(G)$ is finite. Hence by [6. Theorem 17.2] $\mathrm{Z}_{2}(G) / \mathrm{Z}(G)$ is a direct sum of cyclic groups. Let $G / \mathrm{Z}_{2}(G)$ be generated by $x_{1} \mathrm{Z}_{2}(G), \ldots, x_{r} \mathrm{Z}_{2}(G)$ and $H:=\left\langle x_{1}, \ldots, x_{r}\right\rangle$. Then it follows that modulo $\mathrm{Z}(G), H \cap \mathrm{Z}_{2}(G)$ is finite. Thus we can write

$$
\mathrm{Z}_{2}(G) / \mathrm{Z}(G)=\left\langle y_{1} \mathrm{Z}(G)\right\rangle \times \cdots \times\left\langle y_{s} \mathrm{Z}(G)\right\rangle \times\left\langle y_{s+1} \mathrm{Z}(G)\right\rangle \times \cdots
$$

such that $\left(H \cap \mathrm{Z}_{2}(G)\right) \mathrm{Z}(G) / \mathrm{Z}(G) \leq\left\langle y_{1} \mathrm{Z}(G)\right\rangle \times \cdots \times\left\langle y_{s} \mathrm{Z}(G)\right\rangle$. It now follows that the infinite abelian group $\left\langle y_{s+1} \mathrm{Z}(G)\right\rangle \times \cdots$ is a direct factor of $G / \mathrm{Z}(G)$, and the proof is complete.

We are now ready to prove Theorem B.

Proof of Theorem B. Since $\gamma_{2}(G)$ is finite, it follows from Theorem 2.1 that $G / \mathrm{Z}_{2}(G)$ is finite. Now using the fact that $\mathrm{Z}_{2}(G) / \mathrm{Z}\left(\mathrm{Z}_{2}(G)\right)$ is finitely generated, it follows that $G / \mathrm{Z}\left(\mathrm{Z}_{2}(G)\right)$ is finitely generated. Take $\mathrm{Z}\left(\mathrm{Z}_{2}(G)\right)=A$. Then notice that $A$ is a normal abelian subgroup of $G$ such that the index of $C_{G}(A)$ in $G$ is finite, since $\mathrm{Z}_{2}(G) \leq C_{G}(A)$. Hence by Lemma 1.1. $G / \mathrm{Z}(G)$ is finite, which proves (i).

Again take $\mathrm{Z}\left(\mathrm{Z}_{2}(G)\right)=A$ and notice that $\mathrm{Z}_{2}(G) \gamma_{2}(G) \leq \mathrm{C}_{G}(A)$. (ii) now directly follows from Lemma 1.1. If $\mathrm{Z}_{2}(G) \leq \gamma_{2}(G)$, then $\mathrm{Z}_{2}(G)$ is abelian. Thus (iii) follows from (i). Finally, (iv) follows from Proposition 2.2. This completes the proof of the theorem.

We conclude this section with an extension of Theorem A in terms of conjugacy classpreserving automorphisms of given group $G$. An automorphism $\alpha$ of an arbitrary group $G$ is called (conjugacy) class-preserving if $\alpha(g) \in g^{G}$ for all $g \in G$. We denote the group of all class-preserving automorphisms of $G$ by $\operatorname{Aut}_{c}(G)$. Notice that $\operatorname{Inn}(G)$, the group of all inner automorphisms of $G$, is a normal subgroup of $\operatorname{Aut}_{c}(G)$ and $\operatorname{Aut}_{c}(G)$ acts trivially on the center of $G$. A detailed survey on class-preserving automorphisms of finite $p$-groups can be found in 25 . 
Let $G$ be the group as in the statement of Theorem A. Then $G$ is generated by $x_{1}, x_{2}, \ldots, x_{t}$ along with $\mathrm{Z}(G)$. Since $\operatorname{Aut}_{c}(G)$ acts trivially on the center of $G$, it follows that

$$
\left|\operatorname{Aut}_{c}(G)\right| \leq \Pi_{i=1}^{t}\left|x_{i}^{G}\right|
$$

as there are only $\left|x_{i}^{G}\right|$ choices for the image of each $x_{i}$ under any class-preserving automorphism. Since $\left|x_{i}^{G}\right|$ is finite for each $x_{i}, 1 \leq i \leq t$, it follows that $\left|\operatorname{Aut}_{c}(G)\right| \leq \Pi_{i=1}^{t}\left|x_{i}^{G}\right|$ is finite.

We have proved the following result of which Theorem A is a corollary, because $|G / \mathrm{Z}(G)|=$ $|\operatorname{Inn}(G)| \leq\left|\operatorname{Aut}_{c}(G)\right|$

Theorem 2.4. Let $G$ be an arbitrary group such that $G / \mathrm{Z}(G)$ is finitely generated by $x_{1} \mathrm{Z}(G)$, $x_{2} \mathrm{Z}(G), \ldots, x_{t} \mathrm{Z}(G)$ and the conjugacy class of $x_{i}$ in $G$ is of finite length for $1 \leq i \leq t$. Then $\operatorname{Aut}_{c}(G)$ is finite. Moreover $\left|\operatorname{Aut}_{c}(G)\right| \leq \Pi_{i=1}^{t}\left|x_{i}^{G}\right|$ and $\gamma_{2}(G)$ is finite.

Proof of Theorem A is also reproduced using $I A$-automorphisms (automorphisms of a group that induce identity on the abelianization) in [7, Theorem 2.3]. Proof goes on the same way as in the case of class-preserving automorphisms.

\section{Groups with maximal Central quotient}

We start with the following concept due to Hall [8]. For a group $X$, the commutator map $a_{X}: X / \mathrm{Z}(X) \times X / \mathrm{Z}(X) \rightarrow \gamma_{2}(X)$ given by $a_{X}\left(x_{1} \mathrm{Z}(X), x_{2} \mathrm{Z}(X)\right)=\left[x_{1}, x_{2}\right]$ is well defined. Two groups $K$ and $H$ are said to be isoclinic if there exists an isomorphism $\phi$ of the factor group $\bar{K}=K / \mathrm{Z}(K)$ onto $\bar{H}=H / \mathrm{Z}(H)$, and an isomorphism $\theta$ of the subgroup $\gamma_{2}(K)$ onto $\gamma_{2}(H)$ such that the following diagram is commutative

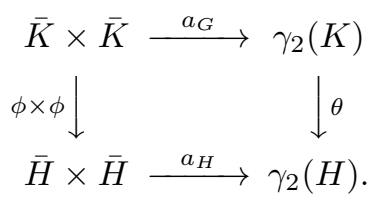

The resulting pair $(\phi, \theta)$ is called an isoclinism of $K$ onto $H$. Notice that isoclinism is an equivalence relation among groups.

The following proposition (also see Macdonald's result [14, Lemma 2.1]) is important for the rest of this section.

Proposition 3.1. Let $G$ be a group such that $G / \mathrm{Z}(G)$ is finite. Then there exists a finite group $H$ isoclinic to the group $G$ such that $\mathrm{Z}(H) \leq \gamma_{2}(H)$. Moreover if $G$ is a p-group, then $H$ is also a p-group.

Proof. Let $G$ be the given group. Then by Schur's theorem $\gamma_{2}(G)$ is finite. Now it follows from a result of Hall [8] that there exists a group $H$ which is isoclinic to $G$ and $\mathrm{Z}(H) \leq \gamma_{2}(H)$. Since $\left|\gamma_{2}(G)\right|=\left|\gamma_{2}(H)\right|$ is finite, $\mathrm{Z}(H)$ is finite. Hence, by the definition of isoclinism, $H$ is finite. Now suppose that $G$ is a $p$-groups. Then it follows that $H / \mathrm{Z}(H)$ as well as $\gamma_{2}(H)$ are $p$-groups. Since $\mathrm{Z}(H) \leq \gamma_{2}(H)$, this implies that $H$ is a $p$-group.

For an arbitrary group $G$ with finite $G / \mathrm{Z}(G)$, we have

$$
|G / \mathrm{Z}(G)| \leq\left|\gamma_{2}(G)\right|^{d}
$$

where $d=d(G / \mathrm{Z}(G))$. For simplicity we say that a group $G$ has Property $A$ if $G / \mathrm{Z}(G)$ is finite and equality holds in (3.2) for $G$. We are now going to classify, upto isoclinism, all groups $G$ having Property A. 
Let $G$ be an arbitrary group having Property A. Then by Proposition 3.1 there exists a finite group $H$ isoclinic to $G$ and, by the definition of isoclinism, $H$ has Property A. Thus for classifying all groups $G$, upto isoclinism, having Property A, it is sufficient to classify all finite group with this property.

Let us first consider non-nilpotent finite groups. For such group we prove the following result in [5]

Theorem 3.3. There is no non-nilpotent group $G$ having Property A.

So we only need to consider finite nilpotent groups. Since a finite nilpotent group is a direct product of it's Sylow $p$-subgroups, it is sufficient to classify finite $p$-groups admitting Property A. Obviously, all abelian groups admit Property A. Perhaps the simplest examples of non-abelian groups having Property A are finite extraspecial $p$-groups. The class of 2 -generated finite capable nilpotent groups with cyclic commutator subgroup also admits Property A. A group $G$ is said to be capable if there exists a group $H$ such that $G \cong H / \mathrm{Z}(H)$. Isaacs [13, Theorem 2] proved: Let $G$ be finite and capable, and suppose that $\gamma_{2}(G)$ is cyclic and that all elements of order 4 in $\gamma_{2}(G)$ are central in $G$. Then $|G / \mathrm{Z}(G)| \leq\left|\gamma_{2}(G)\right|^{2}$, and equality holds if $G$ is nilpotent. So $G$ admits Property A, if $G$ is a nilpotent group as in this statement and $G$ is 2-generated. A complete classification of 2-generated finite capable $p$-groups of class 2 is given in [15].

Motivated by finite extraspecial $p$-groups, a more general class of groups $G$ admitting Property A can be constructed as follows. For any positive integer $m$, let $G_{1}, G_{2}, \ldots, G_{m}$ be 2 -generated finite $p$-groups such that $\gamma_{2}\left(G_{i}\right)=\mathrm{Z}\left(G_{i}\right) \cong X$ (say) is cyclic of order $q$ for $1 \leq i \leq m$, where $q$ is some power of $p$. Consider the central product

$$
Y=G_{1} *_{X} G_{2} *_{X} \cdots *_{X} G_{m}
$$

of $G_{1}, G_{2}, \ldots, G_{m}$ amalgamated at $X$ (isomorphic to cyclic commutator subgroups $\gamma_{2}\left(G_{i}\right), 1 \leq$ $i \leq m)$. Then $|Y|=q^{2 m+1}$ and $|Y / \mathrm{Z}(Y)|=q^{2 m}=\left|\gamma_{2}(Y)\right|^{d(Y)}$, where $d(Y)=2 m$ is the number of elements in any minimal generating set for $Y$. Thus $Y$ has Property A. Notice that in all of the above examples, the commutator subgroup is cyclic. Infinite groups having Property A can be easily obtained by taking a direct product of an infinite abelian group with any finite group having Property A.

We now proceed to showing that any finite $p$-group $G$ of class 2 having Property A is isoclinic to a group $Y$ defined in (3.4).

Let $x \in \mathrm{Z}_{2}(G)$ for a group $G$. Then, notice that $[x, G]$ is a central subgroup of $G$. We have the following simple but useful result.

Lemma 3.5. Let $G$ be an arbitrary group such that $\mathrm{Z}_{2}(G) / \mathrm{Z}(G)$ is finitely generated by $x_{1} \mathrm{Z}(G)$, $x_{2} \mathrm{Z}(G), \ldots, x_{t} \mathrm{Z}(G)$ such that $\exp \left(\left[x_{i}, G\right]\right)$ is finite for $1 \leq i \leq t$. Then

$$
\left|\mathrm{Z}_{2}(G) / \mathrm{Z}(G)\right|=\prod_{i=1}^{t} \exp \left(\left[x_{i}, G\right]\right) .
$$

Proof. By the given hypothesis $\exp \left(\left[x_{i}, G\right]\right)$ is finite for all $i$ such that $1 \leq i \leq t$. Suppose that $\exp \left(\left[x_{i}, G\right]\right)=n_{i}$. Since $\left[x_{i}, G\right] \subseteq \mathrm{Z}(G)$, it follows that $\left[x_{i}^{n_{i}}, G\right]=\left[x_{i}, G\right]^{n_{i}}=1$. Thus $x_{i}^{n_{i}} \in \mathrm{Z}(G)$ and no smaller power of $x_{i}$ than $n_{i}$ can lie in $\mathrm{Z}(G)$, which implies that the order of $x_{i} \mathrm{Z}(G)$ is $n_{i}$. Since $\mathrm{Z}_{2}(G) / \mathrm{Z}(G)$ is abelian, we have $\left|\mathrm{Z}_{2}(G) / \mathrm{Z}(G)\right|=\prod_{i=1}^{t} \exp \left(\left[x_{i}, G\right]\right)$. 
Let $\Phi(X)$ denote the Frattini subgroup of a group $X$. The following result provides some structural information of $p$-groups of class 2 admitting Property A.

Proposition 3.6. Let $H$ be a finite p-group of class 2 having Property $A$ and $\mathrm{Z}(H)=\gamma_{2}(H)$. Then

(i) $\gamma_{2}(H)$ is cyclic;

(ii) $H / \mathrm{Z}(H)$ is homocyclic;

(iii) $[x, H]=\gamma_{2}(H)$ for all $x \in H-\Phi(H)$;

(iv) $H$ is minimally generated by even number of elements.

Proof. Let $H$ be the group given in the statement, which is minimally generated by $d$ elements $x_{1}, x_{2} \ldots, x_{d}$ (say). Since $\mathrm{Z}(H)=\gamma_{2}(H)$, it follows that $H / \mathrm{Z}(H)$ is minimally generated by $x_{1} \mathrm{Z}(H), x_{2} \mathrm{Z}(H), \ldots, x_{d} \mathrm{Z}(H)$. Thus by the identity $|H / \mathrm{Z}(H)|=\left|\gamma_{2}(H)\right|^{d}$, it follows that order of $x_{i} \mathrm{Z}(H)$ is equal to $\left|\gamma_{2}(H)\right|$ for all $1 \leq i \leq d$. Since the exponent of $H / \mathrm{Z}(H)$ is equal to the exponent of $\gamma_{2}(H)$, we have that $\gamma_{2}(H)$ is cyclic and $H / \mathrm{Z}(H)$ is homocyclic. Now by Lemma 3.5. $\left|\gamma_{2}(H)\right|^{d}=|H / \mathrm{Z}(H)|=\prod_{i=1}^{t} \exp \left(\left[x_{i}, H\right]\right)$. Since $\left[x_{i}, H\right] \subseteq \gamma_{2}(H)$, this implies that $\left[x_{i}, H\right]=\gamma_{2}(H)$ for each $i$ such that $1 \leq i \leq d$. Let $x$ be an arbitrary element in $H-\Phi(H)$. Then the set $\{x\}$ can always be extended to a minimal generating set of $H$. Thus it follows that $[x, H]=\gamma_{2}(H)$ for all $x \in H-\Phi(H)$. This proves first three assertions.

For the proof of (iv), we consider the group $\bar{H}=H / \Phi\left(\gamma_{2}(H)\right)$. Notice that both $H$ as well as $\bar{H}$ are minimallay generated by $d$ elements. Since $[x, H]=\gamma_{2}(H)$ for all $x \in H-\Phi(H)$, it follows that for no $x \in H-\Phi(H), \bar{x} \in \mathrm{Z}(\bar{H})$, where $\bar{x}=x \Phi\left(\gamma_{2}(H)\right) \in \bar{H}$. Thus it follows that $\mathrm{Z}(\bar{H}) \leq \Phi(\bar{H})$. Also, since $\gamma_{2}(H)$ is cyclic, $\gamma_{2}(\bar{H})$ is cyclic of order $p$. Thus it follows that $\bar{H}$ is isoclinic to a finite extraspecial $p$-group, and therefore it is minimally generated by even number of elements. Hence $H$ is also minimally generated by even number of elements. This completes the proof of the proposition.

Using the definition of isoclinism, we have

Corollary 3.7. Let $G$ be a finite p-group of class 2 admitting Property A. Then $\gamma_{2}(G)$ is cyclic and $G / \mathrm{Z}(G)$ is homocyclic.

We need the following important result.

Theorem 3.8 ([3], Theorem 2.1). Let $G$ be a finite p-group of nilpotency class 2 with cyclic center. Then $G$ is a central product either of two generator subgroups with cyclic center or two generator subgroups with cyclic center and a cyclic subgroup.

Theorem 3.9. Let $G$ be a finite p-group of class 2 having Property A. Then $G$ is isoclinic to the group $Y$, defined in (3.4), for suitable positive integers $m$ and $n$.

Proof. Let $G$ be a group as in the statement. Then by Proposition 3.1 there exists a finite $p$-group $H$ isoclinic to $G$ such that $\mathrm{Z}(H)=\gamma_{2}(H)$. Obviously $H$ also satisfies $|H / \mathrm{Z}(H)|=\left|\gamma_{2}(H)\right|^{d}$, where $d$ denotes the number of elements in any minimal generating set of $G / \mathrm{Z}(G)$. Then by Proposition 3.6, $\gamma_{2}(H)=\mathrm{Z}(H)$ is cyclic of order $q=p^{n}$ (say) for some positive integer $n$, and $H / \mathrm{Z}(H)$ is homocyclic of exponent $q$ and is of order $q^{2 m}$ for some positive integer $m$. Since $\mathrm{Z}(H)=\gamma_{2}(H)$ is cyclic, it follows from Theorem 3.8 that $H$ is a central product of 2-generated 
groups $H_{1}, H_{2}, \ldots, H_{m}$. It is easy to see that $\gamma_{2}\left(H_{i}\right)=\mathrm{Z}\left(H_{i}\right)$ for $1 \leq i \leq m$ and $\left|\gamma_{2}(H)\right|=q$. This completes the proof of the theorem.

We would like to remark that Theorem [3.9] is also obtained in [26. Theorem 11.2] as a consequence on study of class-preserving automorphisms of finite $p$-group. But we have presented here a direct proof.

Now we classify finite $p$-groups of nilpoency class larger than 2 . Consider the metacylic groups

$$
K:=\left\langle x, y \mid x^{p^{r+t}}=1, y^{p^{r}}=x^{p^{r+s}},[x, y]=x^{p^{t}}\right\rangle,
$$

where $1 \leq t<r$ and $0 \leq s \leq t(t \geq 2$ if $p=2)$ are non-negative integers. Notice that the nilpotency class of $K$ is at least 3. Since $K$ is generated by 2 elements, it follows from (2.3) that $\left|\operatorname{Aut}_{c}(K)\right| \leq\left|\gamma_{2}(K)\right|^{2}=p^{2 r}$. It is not so difficult to see that $|\operatorname{Inn}(K)|=|K / \mathrm{Z}(K)|=p^{2 r}$. Since $\operatorname{Inn}(K) \leq \operatorname{Aut}_{c}(K)$, it follows that $\left|\operatorname{Aut}_{c}(K)\right|=|\operatorname{Inn}(K)|=\left|\gamma_{2}(K)\right|^{2}=\left|\gamma_{2}(K)\right|^{d(K)}$ (That $\operatorname{Aut}_{c}(G)=\operatorname{Inn}(G)$, is, in fact, true for all finite metacylic $p$-groups). Thus $K$ admits Property A. Furthermore, if $H$ is any 2-generator group isoclinic to $K$, then it follows that $H$ admits Property A. For a finite $p$-groups having Property A, there always exists a $p$-group $H$ isoclinic $G$ such that $|H / \mathrm{Z}(H)|=\left|\gamma_{2}(H)\right|^{d}$, where $d=d(H)$. The following theorem now classifies, upto isoclinism, all finite $p$-groups $G$ of nilpotency class larger than 2 having Property A.

Theorem 3.11 (Theorem 11.3, [26]). Let $G$ be a finite p-group of nilpotency class at least 3. Then the following hold true.

(i) If $|G / \mathrm{Z}(G)|=\left|\gamma_{2}(G)\right|^{d}$, where $d=d(G)$, then $d(G)=2$;

(ii) If $\left|\gamma_{2}(G) / \gamma_{3}(G)\right|>2$, then $|G / \mathrm{Z}(G)|=\left|\gamma_{2}(G)\right|^{d}$ if and only if $G$ is a 2-generator group with cyclic commutator subgroup. Furthermore, $G$ is isoclinic to the group $K$ defined in (3.10) for suitable parameters;

(iii) If $\left|\gamma_{2}(G) / \gamma_{3}(G)\right|=2$, then $|G / \mathrm{Z}(G)|=\left|\gamma_{2}(G)\right|^{d}$ if and only if $G$ is a 2-generator 2-group of nilpotency class 3 with elementary abelian $\gamma_{2}(G)$ of order 4 .

It is clear that the groups $G$ occuring in Theorem 3.11(iii) are isoclinic to certain groups of order 32. Using Magma (or GAP), one can easily show that such groups of order 32 are SmallGroup $(32, \mathrm{k})$ for $k=6,7,8$ in the small group library.

We conclude this section with providing some different type of bounds on the central quotient of a given group. If $\left|\gamma_{2}(G) \mathrm{Z}(G) / \mathrm{Z}(G)\right|=n$ is finite for a group $G$, then it follows from [22, Theorem 1] that $\left|G / \mathrm{Z}_{2}(G)\right| \leq n^{2 \log _{2} n}$. Using this and Lemma 3.5 we can also provide an upper bound on the size of $G / \mathrm{Z}(G)$ in terms of $n$, the rank of $\mathrm{Z}_{2}(G) / \mathrm{Z}(G)$ and exponents of certain sets of commutators (here these sets are really subgroups of $G$ ) of coset representatives of generators of $\mathrm{Z}_{2}(G) / \mathrm{Z}(G)$ with the elements of $G$. This is given in the following theorem.

Theorem 3.12. Let $G$ be an arbitrary group. Let $\left|\gamma_{2}(G) \mathrm{Z}(G) / \mathrm{Z}(G)\right|=n$ is finite and $\mathrm{Z}_{2}(G) / \mathrm{Z}(G)$ is finitely generated by $x_{1} \mathrm{Z}(G), x_{2} \mathrm{Z}(G), \ldots, x_{t} \mathrm{Z}(G)$ such that $\exp \left(\left[x_{i}, G\right]\right)$ is finite for $1 \leq i \leq t$. Then

$$
|G / \mathrm{Z}(G)| \leq n^{2 \log _{2} n} \prod_{i=1}^{t} \exp \left(\left[x_{i}, G\right]\right)
$$

\section{Problems AND Examples}

Theorem B provides some conditions on a group $G$ under which $G / \mathrm{Z}(G)$ becomes finite. It is interesting to solve 
Problem 1. Let $G$ be an arbitrary group. Provide a set $\mathcal{C}$ of optimal conditions on $G$ such that $G / \mathrm{Z}(G)$ is finite if and only if all conditions in $\mathcal{C}$ hold true.

As we know that there is no finite non-nilpotent group $G$ admitting Property A. Since $\operatorname{Inn}(G) \leq \operatorname{Aut}_{c}(G)$, it is interesting to consider

Problem 2. Classify all non-nilpotent finite group $G$ such that $\left|\operatorname{Aut}_{c}(G)\right|=\left|\gamma_{2}(G)\right|^{d}$, where $d=d(G)$.

A much stronger result than Theorem 3.3 is known in the case when the Frattini subgroup of $G$ is trivial. This is given in the following theorem of Herzog, Kaplan and Lev 10, Theorem A] (the same result is also proved independently by Halasi and Podoski in [11, Theorem 1.1]).

Theorem 4.1. Let $G$ be any non-abelian group with trivial Frattini subgroup. Then $|G / \mathrm{Z}(G)|<$ $\left|\gamma_{2}(G)\right|^{2}$.

The following result with the assertion similar to the preceding theorem is due to Isaacs [13].

Theorem 4.2. If $G$ is a capable finite group with cyclic $\gamma_{2}(G)$ and all elements of order 4 in $\gamma_{2}(G)$ are central in $G$, then $|G: Z(G)| \leq\left|\gamma_{2}(G)\right|^{2}$. Moreover, equality holds if $G$ is nilpotent.

So, there do exist nilpotent groups with comparatively small central quotient. A natural problem is the following.

Problem 3. Classify all finite $p$-groups $G$ such that $|G: Z(G)| \leq\left|\gamma_{2}(G)\right|^{2}$.

Let $G$ be a finite nilpotent group of class 2 minimally generated by $d$ elements. Then it follows from Lemma 3.5 that $|G / \mathrm{Z}(G)| \leq \prod_{i=1}^{d} \exp \left(\left[x_{i}, G\right]\right)$, which in turn implies

$$
|G / \mathrm{Z}(G)| \leq\left|\exp \left(\gamma_{2}(G)\right)\right|^{d} .
$$

Problem 4. Classify all finite $p$-groups $G$ of nilpotency class 2 for which equality holds in (4.3).

Now we discuss some examples of infinite groups with finite central quotient. The most obvious example is the infinite cyclic group. Other obvious examples are the groups $G=H \times \mathcal{Z}$, where $H$ is any finite group and $\mathcal{Z}$ is the infinite cyclic group. Non-obvious examples include finitely generated $F C$-groups, in which conjugacy class sizes are bounded, and certain Cernikov groups. We provide explicit examples in each case. Let $F_{n}$ be the free group on $n$ symbols and $p$ be a prime integer. Then the factor group $F_{n} /\left(\gamma_{2}\left(F_{n}\right)^{p} \gamma_{3}\left(F_{n}\right)\right)$ is the required group of the first type, where $\gamma_{2}\left(F_{n}\right)^{p}=\left\langle u^{p} \mid u \in \gamma_{2}\left(F_{n}\right)\right\rangle$. Now let $H=\mathcal{Z}\left(p^{\infty}\right) \times A$ be the direct product of quasi-cyclic (Prüfer) group $\mathcal{Z}\left(p^{\infty}\right)$ and the cyclic group $A=\langle a\rangle$ of order $p$, where $p$ is a prime integer. Now consider the group $G=H \rtimes B$, the semidirect product of $H$ and the cyclic group $B=\langle b\rangle$ of order $p$ with the action by $x^{b}=x$ for all $x \in \mathcal{Z}\left(p^{\infty}\right)$ and $a^{b}=a c$, where $c$ is the unique element of order $p$ in $\mathcal{Z}\left(p^{\infty}\right)$. This group is suggested by V. Romankov and Rahul D. Kitture through ResearchGate, and is a Cernikov group.

The following problem was suggested by R. Baer in [1].

Problem 5. Let $A$ be an abelian group and $Q$ be a group. Obtain necessary and sufficient conditions on $\mathrm{A}$ and $G$ so that there exists a group $Q$ with $A \cong \mathrm{Z}(G)$ and $Q \cong G / A$. 
This problem was solved by Baer himself for an arbitrary abelian group A and finitely generated abelian group G. Moskalenko [16] solved this problem for an arbitrary abelian group A and a periodic abelian group G. He [17] also solved this problem for arbitrary abelian group A and a non-periodic abelian group $\mathrm{G}$ such that the rank of $G / t(G)$ is 1 , where $t(G)$ denotes the tortion subgroup of $G$. If this rank is more than 1 , then he solved the problem when $A$ is a torsion abelian group. For a given group $Q$, the existence of a group $G$ such that $Q \cong G / \mathrm{Z}(G)$ has been studied extensively under the theme 'Capable groups'. However, to the best of our knowledge, Problem 5 has been poorly studied in full generality. Let us restate a special case of this problem in a little different setup. A pair of groups $(A, Q)$, where $A$ is an arbitray abelian group and $Q$ is an arbitrary group, is said to be a capable pair if there exists a group $G$ such that $A \cong \mathrm{Z}(G)$ and $Q \cong G / \mathrm{Z}(G)$. So, in our situation, the following problem is very interesting.

Problem 6. Classify capable pairs $(A, Q)$, where $A$ is an infinite abelian group and $Q$ is a finite group.

Finally let us get back to the situation when $G$ is a group with finite $\gamma_{2}(G)$ but infinite $G / \mathrm{Z}(G)$. The well known examples of such type are infinite extraspecial $p$-groups. Other class of examples can be obtained by taking a central product (amalgamated at their centers) of infinitely many copies of a 2-generated finite $p$-group of class 2 such that $\gamma_{2}(H)=\mathrm{Z}(H)$ is cyclic of order $q$, where $q$ is some power of $p$. Notice that both of these classes consist of groups of nilpotency class 2. Now if we take $G=X \times H$, where $X$ is an arbitrary group with finite $\gamma_{2}(X)$ and $H$ is with finite $\gamma_{2}(H)$ and infinite $H / \mathrm{Z}(H)$, then $\gamma_{2}(G)$ is finite but $G / \mathrm{Z}(G)$ is infinite. So we can construct nilpotent groups of arbitrary class and even non-nilpotent group with infinite central quotient and finite commutator subgroup.

A non-nilpotent group $G$ is said to be purely non-nilpotent if it does not have any non-trivial nilpotent subgroup as a direct factor. With the help of Rahul D. Kitture, we have also been able to construct purely non-nilpotent groups $G$ such that $\gamma_{2}(G)$ is finite but $G / \mathrm{Z}(G)$ is infinite. Let $H$ be an infinite extra-special $p$-group. Then we can always find a field $\mathcal{F}_{q}$, where $q$ is some power of a prime, containing all $p$ th roots of unity. Now let $K$ be the special linear group $s l\left(p, \mathcal{F}_{q}\right)$, which is a non-nilpotent group having a central subgroup of order $p$. Now consider the group $G$ which is a central product of $H$ and $K$ amalgamated at $\mathrm{Z}(H)$. Then $G$ is a purely non-nilpotent group with the required conditions. It will be interesting to see more examples of this type which do not occur as a central product of such infinite groups of nilpotency class 2 with non-nilpotent groups.

By Proposition 2.2 we know that for an arbitrary group $G$ with finite $\gamma_{2}(G)$ but infinite $G / \mathrm{Z}(G)$, the group $G / \mathrm{Z}(G)$ has an infinite abelian group as a direct factor. Further structural information is highly welcome.

Problem 7. Provide structural information of the group $G$ such that $\gamma_{2}(G)$ is finite but $G / \mathrm{Z}(G)$ is infinite?

\section{REFERENCES}

[1] R. Baer, Groups with preassigned central and central quotient group, Trans. Amer. Math. Soc. 44 (1938), $387-412$.

[2] W. Bosma, J. Cannon and C. Playoust, The Magma algebra system. I. The user language, J. Symbolic Comput., 24 (1997), 235-265. 
[3] J. M. Brady, R. A. Bryce, and J. Cossey, On certain abelian-by-nilpotent varieties, Bull. Austral. Math. Soc. 1 (1969), 403 - 416.

[4] Y. Cheng, On finite p-groups with cyclic commutator subgroup, Arch. Math. 39 (1982), 295-298.

[5] S. Dolfi, E. Pacifici and M. K. Yadav, Bounds for the index of the centre in non-nilpotent groups, preprint.

[6] L. Fuchs, Infinite abelian groups, Vol. I Pure and Applied Mathematics, Vol 36 Academic Press, Yew York - London, 1970.

[7] D. K. Gumber and H. Kalra, On the converse of a theorem of Schur, Arch. Math. (Basel) 101 (2013), 17-20.

[8] P. Hall, The classification of prime power groups, Journal für die reine und angewandte Mathematik 182 (1940), 130-141.

[9] P. Hall, Finite-by-nilpotent groups, Proc. Cambridge Phil. Soc. 52 (1956), 611-616.

[10] M. Herzog, G. Kaplan and A. Lev, The size of the commutator subgroup of finite groups, J. Algebra 320 (2008), 980-986.

[11] Z. Halasi and K. Podoski, Bounds in groups with trivial Frattini subgroups, J. Algebra 319 (2008), 893-896.

[12] P. Hilton, On a theorem of Schur, Int. J. Math. Math. Sci. 28 (2001), 455-460.

[13] I. M. Isaacs, Derived subgroups and centers of capable groups, Proc. Amer. Math. Soc. 129 (2001), 2853-2859.

[14] I. D. Macdonald, Some explicit bounds in groups with finite derived groups, Proc. London Math. Soc. (3) 11 (1961), 23-56.

[15] A. Magidin and R. F. Morse, Certain homological functors of 2-generator p-groups of class 2, Contemp. Math. 511 (2010), 127-166.

[16] A. I. Moskalenko, Central extensions of abelian groups by means of abelian groups, Sibirsk. Mat. Z. 9 (1968), 104-115.

[17] A. I. Moskalenko, Central extensions of a periodic abelian group by means of an abelian group. Algebra and number theory. Moskov. Gos. Ped. Inst. Ucen. Zap. 375 (1971), 80-84.

[18] B. H. Neumann, Groups with finite classes of conjugate elements, Proc. London Math. Soc. (3) 1 (1951), 178-187.

[19] B. H. Neumann, Groups covered by permutable subsets, J. London Math. Soc. 29 (1954), 236-248.

[20] B. H. Neumann, A problem of Paul Erdös on groups, J. Austral. Math. Soc. 21 (Series A) (1976), 467-472.

[21] P. Niroomand, The converse of Schur's theorem, Arch. Math. 94 (2010), 401-403.

[22] K. Podoski and B. Szegedy, Bounds for the index of the centre in capable groups, Proc. Amer. Math. Soc. 133 (2005), 3441-3445.

[23] I. Schur, Über die Darstellung der endlichen Gruppen durch gebrochene lineare Substitutionen, Journal für die reine und angewandte Mathematik 127 (1904), 20-50.

[24] B. Sury, A generalization of a converse of Schur's theorem, Arch. Math. 95 (2010), 317-318.

[25] M. K. Yadav, Class-preserving automorphisms of finite p-groups: a survey, Groups St Andrews - 2009 (Bath), LMS Lecture Note Ser. 388 (2011), 569-579.

[26] M. K. Yadav, Class-preserving automorphisms of finite p-groups II, Israel J. Math. 209 (2015), 355-396. DOI: $10.1007 / \mathrm{s} 11856-015-1222-4$

School of Mathematics, Harish-Chandra Research Institute, Chhatnag Road, Jhunsi, Allahabad - 211019, INDIA

E-mail address: myadav@hri.res.in 